\title{
Surgery first: Which protocols for which results?
}

\section{Zeitoun}

Qualified Specialist in Dento-facial Orthopedics, Former Hospital Resident

\section{SUMMARY}

The conventional surgical-orthodontic approach starts with a long period of preoperative orthodontic preparation considered by the majority of patients as the most difficult phase of treatment in terms of length, pain but also esthetic and functional discomfort. However, in recent decades, it was noted that the patient's expectations increased with decreased treatment time. Performing surgery first therefore met these requirements by eliminating the preoperative orthodontic phase resulting in shorter treatments and a higher satisfaction rate for the patient's esthetical expectations at the beginning of treatment.

After introducing the protocol, indications, advantages and disadvantages of this technique, we will focus on the treatment outcomes that can be achieved by the surgical-orthodontic team: can this approach be considered as a new golden treatment standard for surgical-orthodontic treatments?

\section{KEYWORDS}

Orthognathic surgery, orthodontics

\section{INTRODUCTION}

The treatment of significant skeletal dysmorphoses in adults requires the intervention of orthognathic surgery in conjunction with orthodontic treatment. Given the improvement in surgical techniques and the increase in the number of patients choosing this type of treatment, patients and clinicians have been looking for therapeutic approaches to obtain optimal esthetic and occlusal results. The conventional orthosurgical approach begins with a long phase of preoperative orthodontic preparation. This step is considered by the majority of patients as the most difficult treatment step in terms of duration and pain, but also esthetic and functional discomfort. Other innovative protocols were then introduced, such as the 'surgery first approach.' What is this protocol? What are its indications, advantages, and disadvantages? Can this approach be considered a therapeutic alternative on the same level as conventional orthosurgical procedures?

Address for correspondence:

Diane Zeitoun

26 rue de Chazelles - 75017 Paris

E-mail: dianezeitoun@hotmail.com

This is an Open Access article distributed under the terms of the Creative Commons Attribution License (http://creativecommons.org/licenses/by/4.0), which permits unrestricted use, distribution, and reproduction in any medium, provided the original work is properly cited. 


\section{HISTORY}

Before 1960, orthognathic surgeries were performed without prior orthodontic preparation, especially for skeletal Class-III corrections. The technique used in the mandible was a vertical osteotomy at the ramus level introduced by Caldwell and Letterman in $1954^{6}$. Bilateral sagittal split ramus osteotomy was described by Trauner and Obwegeser in 1957, marking the beginning of the modern era in orthognathic surgery ${ }^{35}$. As far as the maxilla is concerned, the Lefort I osteotomy was first described in 1859 by Langenbeck ${ }^{20}$ then it was developed during the 1960s to allow the repositioning of the maxilla in the three planes of space ${ }^{4,10}$.

At that time, surgeons did not consider the need for orthodontic preparation before surgery. Orthognathic surgeries used to be performed before orthodontic treatments after the removal of devices or without any orthodontic treatment ${ }^{14,32}$.

The combination of orthodontics and orthognathic surgery began later on with the aim of straightening the anterior teeth on the basal bone before sur- gery to prevent their inclination from limiting surgical movements. Converse and Horowitz in 1969 outlined the need for alignment and coordination of dental arches. Obwegeser felt it was necessary for preoperative orthodontic treatment to be an integral part of the conventional approach to surgical treatment of dentofacial anomalies ${ }^{28}$. Worms et al. ${ }^{42}$ in 1976 supported this concept of "orthodontics first" and even extended it to all surgical cases stressing that the surgical repositioning of the jaws is possible only after the elimination of all dental compensations. The objectives of preoperative orthodontic treatments were then defined: dental decompensation by positioning the teeth on their basal bone, leveling and alignment of the teeth to correct any existing congestion, coordination of the upper and lower arches, divergence of the teeth, and the root divergence where osteotomies were planned $^{41}$. This "orthodontics first" approach then became the standard procedure used by most orthognathic teams after the 1970s.

\section{THE SURGERY FIRST CONCEPT}

In 1988, Behrman and Behrman introduced the concept of "surgery first and orthodontics second" according to the fact that the adult patient has social, economic, and psychological requirements that must be met as early as possible during treatment. They also stated that the "surgery first" procedure facilitates the necessary dental movements and decreases the total duration of orthodontic treatment. Nevertheless, because no valid study could support these principles, few orthodontic teams adopted this concept ${ }^{3}$. In 2007, Dr. Bell and colleagues at the University of Southwest Texas Medical Center organized a symposium on

"Paradigm Shifts in Orthognathic Surgery" ${ }^{5}$. They stated that many facial malformations and malocclusions are 
treated inefficiently over long periods of time by orthognathic surgeries that are too invasive, complicated, expensive, and unpredictable. According to them, the advances and sophistication of orthognathic surgery and orthodontic techniques could speed up treatment and improve the quality of patient care. The 2011 symposium then presented the approach of "surgery first"14.

This protocol corresponds to a modification of the initial protocol by carrying out the surgery before any orthodontic treatment to accelerate the orthodontic phase ${ }^{29}$. The concept of

\section{INDICATIONS FOR SURGERY FIRST}

No special criteria for the use of surgery first have been recorded by the authors. The majority of malocclusion types and dentofacial anomalies have been described as being able to be treated using "surgery first" ${ }^{36}$. Case re-

\section{SURGERY FIRST PROTOCOL (Fig.1)}

In the surgery first protocol, treatment planning must be done with precision. Orthodontic and surgical movements should be previsualized as an occlusion and cannot be used as a guide during surgery to achieve treatment goals ${ }^{26,34}$. The surgeon must perform a precise osteotomy and intermaxillary fixation using gutters on nonaligned dental arches and ensure good stability after repositioning the jaws ${ }^{14}$. After surgery, transient occlusion is established in an appropriate molar relationship and will need to be transformed into a solid final occlusion ${ }^{22}$. this technique lies in the absence of any dental movement beforehand. Surgery is used to establish an appropriate maxillomandibular relationship before orthodontic treatment. This allows the improvement of the facial esthetics from the beginning of the treatment, which is usually the major concern of the patient ${ }^{22}$. The name SFOA (Surgery-First-Orthognathic Approach) or SFA (Surgery First Approach) was introduced by Hyon in 2008, which introduced specific elements and limits, thus changing the paradigm in orthognathic surgery ${ }^{15}$.

ports from surgery first can therefore be found in the literature for skeletal Class II, supraocclusions, gaps, and asymmetries, but the majority are skel-

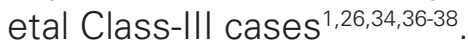

The orthodontist should accurately plan out postoperative orthodontic treatments from the beginning of the preoperative treatment plan including dental alignment, incisive decompensation, and arch coordination and widening. The orthodontist must therefore be aware of the principles and limits of orthodontic movements as well as the principles of orthognathic surgery, to plan the postoperative treatment ${ }^{2}$. To achieve this goal, the surgeon and the orthodontist using a "surgery first" approach must be experienced and 


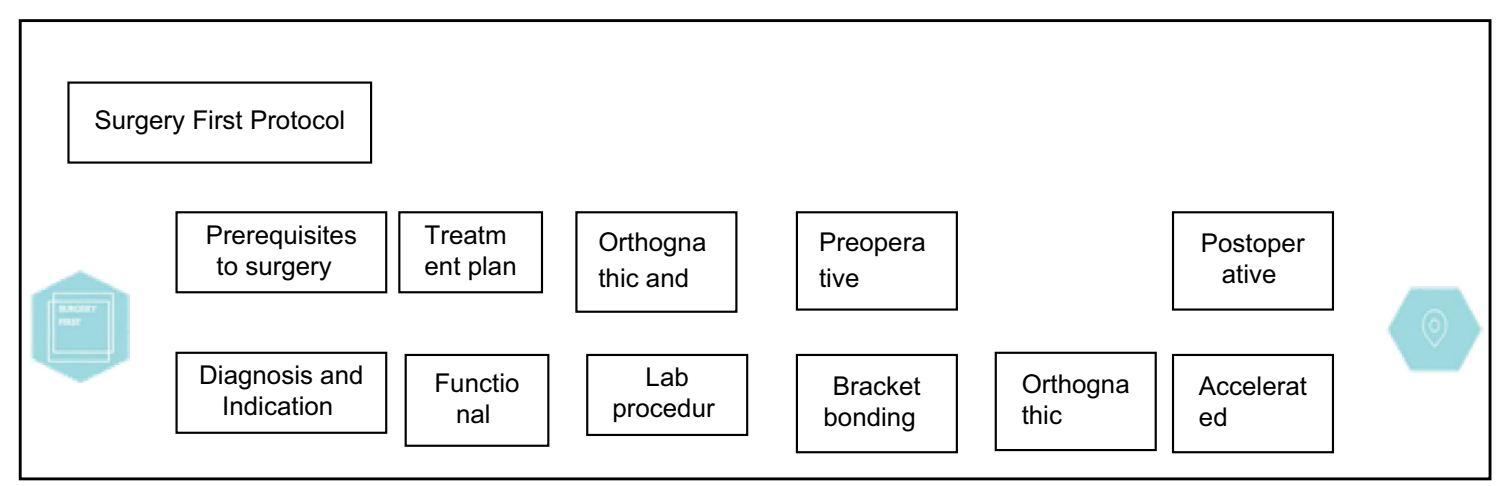

Figure 1

Surgery first protocol.

cooperate closely with each other to obtain predictable and satisfactory results ${ }^{14,17}$.

Models with setups are usually used to predict and simulate the surgical movement of the jaws as well as the dental movements and the coordination of the end-of-treatment arches. Baek et al. ${ }^{2}$ describes a technique using a semiadaptable articulator with a separation of the dental parts and the base of the study models. This allows the setup to separately consider the required dental alignment and skeletal changes. They are then used for the preparation of intermediate and final surgical gutters. Nowadays, some orthognathic teams use virtual planning for orthognathic osteotomies. This virtual 3D planning allows the manufacture of a gutter by computer-aided design (CAD-CAM) to ensure proper jaw positioning (Fig. 2). In the same way, the dental movements needed in future orthodontic treatment can be simulated by a 3D virtual orthodontic setup integrated into the 3D model of the skull ${ }^{11}$.

In the surgery first protocol, orthodontic devices are put in place before surgery. The various authors reported bonding the brackets $24 \mathrm{~h}$ to immedi- ately before $26,34,37$, a week before ${ }^{11,12,18,22}$, 1 month before $18,21,39$ or 1-2 months ${ }^{2}$ before surgery. Only one study reported total elimination of the preoperative orthodontic phase by positioning orthodontic brackets 10-14 days after surgery ${ }^{12}$.

premature occlusal contacts, articles have reported the use of active arches a few weeks before surgery to correct these slight malpositions, this is what Hernandez et al. ${ }^{11}$ call "early surgery."

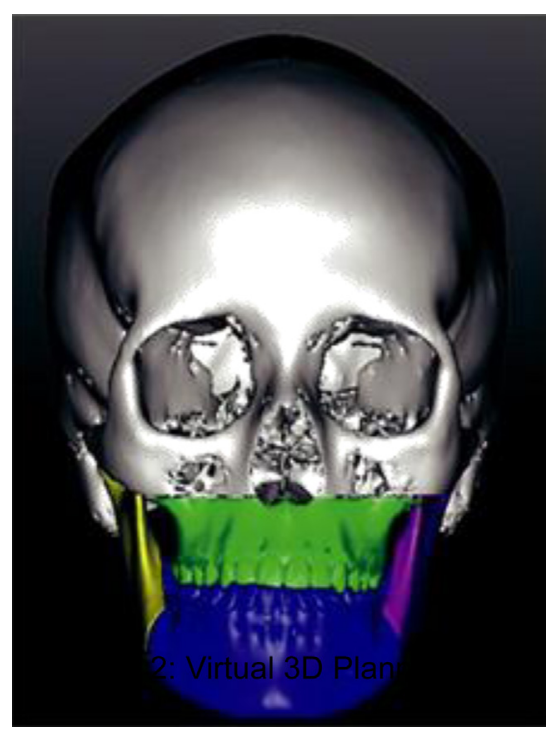

Figure 2

Virtual 3D plan. 


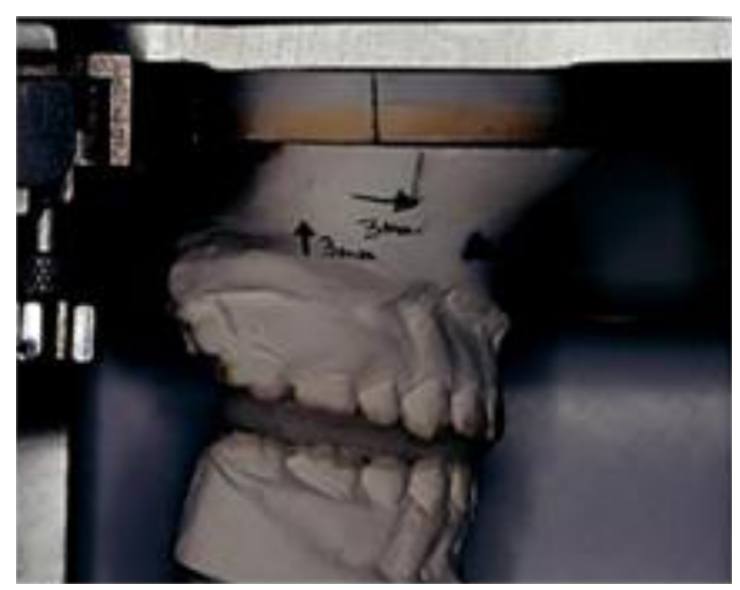

Figure 3

Surgical gutter on models mounted on an articulator.

Surgery is performed with surgical gutters (Fig. 3) and intermaxillary fasteners as guides for the final position of the jaw. Bone plates are then used for the rigid support of the maxillae, but the length of time during which the surgical gutter is put in place

In the case where the presence of congestion creates depends on the orthodontist. Some clinicians only use the gutter during surgery 22,36,38; other authors suggest leaving the gutter in place for 4-6 weeks after surgery for all patients ${ }^{2,16}$ or more specifically in cases where maxillary transverse expansion has been achieved ${ }^{36}$. In their case reports, Nagasaka et al. ${ }^{26}$ and Sugawara et $a .^{34}$ use a removable surgical gutter covering all the incisal edges and/ or occlusal surfaces with a lingual bar and ball hooks to ensure an optimal fit on the two arches. This gutter is then transformed into a removable postoperative occlusal gutter to stabilize the position of the jaws and facilitate masticatory function.

The arch can be placed shortly before $18,21,36,39,43$ or after $2,22,26,34,38$ surgery.
Nevertheless, dental movements that may occur before the surgery could make the adaptation of the individual gutter inaccurate, which would interfere with the correct positioning of the jaws. For this reason, the authors suggested that the first nickel-titanium arch should be placed $24 \mathrm{~h}$ before surgery, the day of surgery, or even at the first postoperative orthodontic appointment taking place 1-2 weeks after the surgery ${ }^{36,38}$. One of the main goals of the postoperative orthodontic phase is to take advantage of the accelerated postoperative orthodontic movement known as rapid acceleratory phenomenon (RAP). This is why most authors have suggested that the arch should be inserted immediately after surgery without gutter or intermaxillary fixation so as to allow free movement of the teeth ${ }^{21,23,37,38}$. In this protocol, patients are seen every 2-4 weeks for follow-ups involving a possible change of arches and control or modification of the force vectors of the intermaxillary elastics when necessary ${ }^{11,12,36-38}$.

On the other hand, case reports $21,26,38$ described the use of the skeletal anchorage system (SAS) within a surgery first protocol. SAS uses titanium miniplates as temporary anchoring devices to achieve predictable tooth movements of all teeth in all three dimensions of space in patients whose growth is complete.

Miniplates are placed during the orthognathic surgery and are used to fix the significant orthodontic displacements often necessary in this type of protocol. Other authors have described the use of miniscrews placed during the first postoperative month. These skeletal anchors prevent the premature loading of dental anchors after surgery and prevent any possible unwanted dental movements ${ }^{12}$. 


\section{WHY SURGERY FIRST? WHAT ARE THE BENEFITS?}

The SFA has several biological and psychosocial advantages compared to conventional orthosurgical treatment. Nagasaka et al. ${ }^{26}$ believes that the benefits of this new treatment approach clearly outweigh its disadvantages, which is why it should become one of the standard therapeutic alternatives in the near future.

First of all, the improvement on the psychology and well-being of the patient is aided through the improvement of their facial esthetics and functions. Functional and esthetic disorders due to dentofacial or skeletal abnormalities with a great psychosocial impact are observed. They can lead to low self-esteem, which can lead to depression. Orthognathic surgery is the only therapy that treats these dysmorphoses ${ }^{29}$. The SFA has a real advantage because it improves the facial esthetics and functions from the beginning of the treatment. The patient does not have to wait many months or even years due to preparatory orthodontic treatment. Because the skeletal problem and therefore the esthetic disorder is corrected,

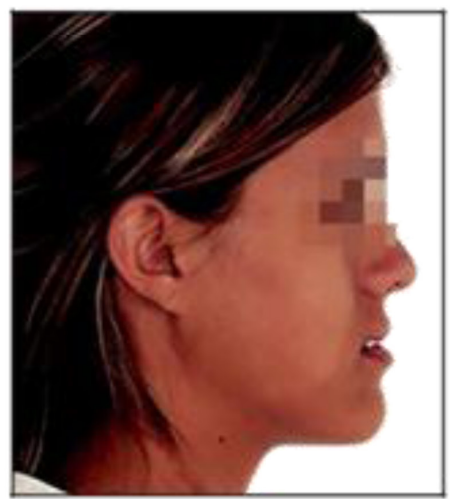

a

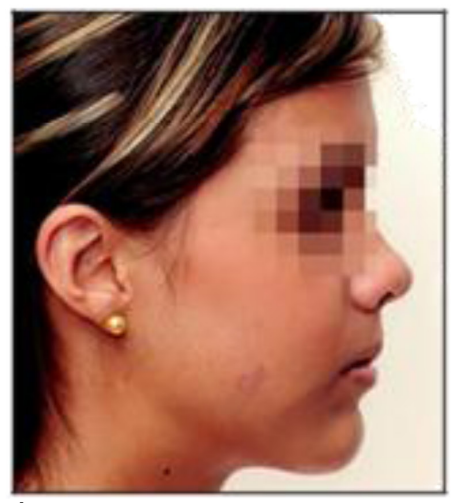

b
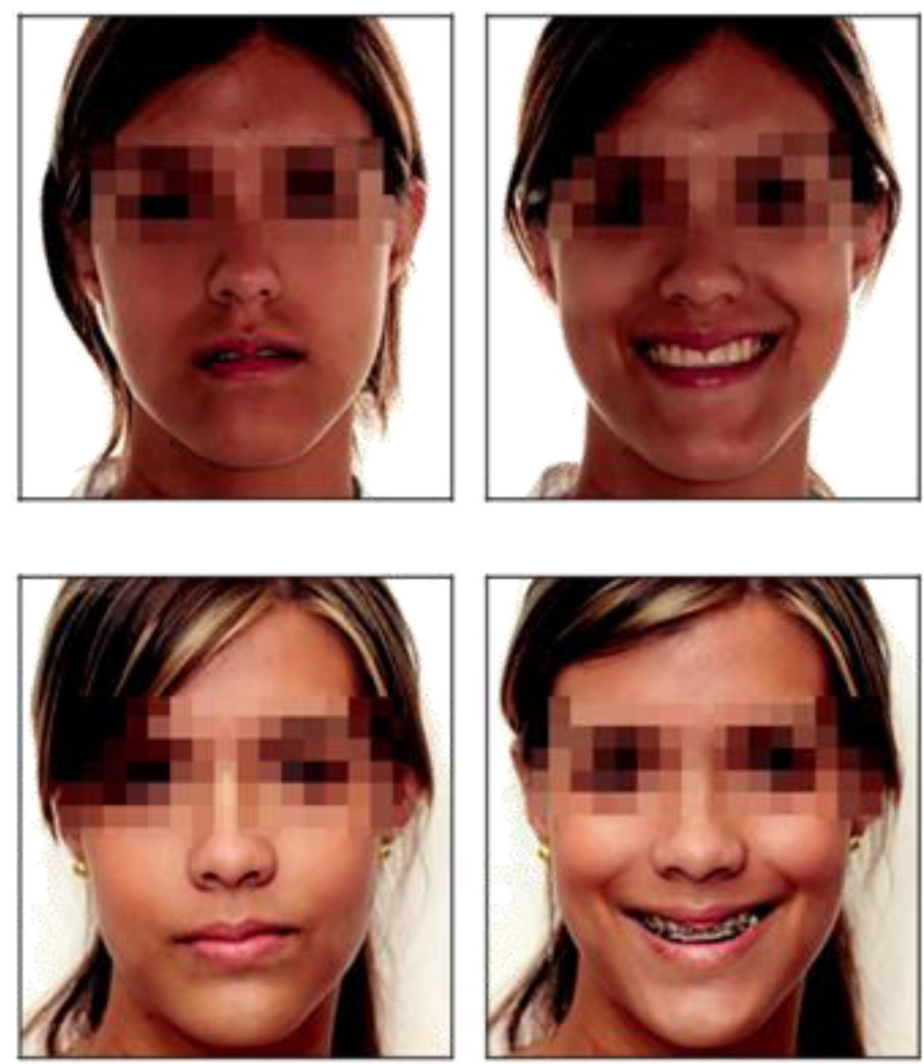

Figure 4

(a) Consultant patient for mandibular prognathism, preoperative photographs. (b) The orthodontic phase has just begun with a very satisfactory esthetic result at 1 month after surgery. 
the patients are satisfied from the beginning of the treatment ${ }^{12,20,37}$ (Fig. 4 a, b). On the other hand, the absence of preoperative dental decompensation is a real advantage because the preoperative orthodontic preparation involves a worsening of the profile especially at the level of the lips, masticatory discomfort, and psychosocial problems. The SFA helps fix the major problems associated with the traditional protocol ${ }^{26,31}$.

As a result, different authors have observed increased patient cooperation treated by SFA compared to a conventional approach. In 2014, Hernandez-Alfaro et al. ${ }^{13}$ stated that early response to patient demand improves adherence to postoperative orthodontic treatment and makes a significant contribution to overall treatment satisfaction. Because of the treatment sequence of a surgery first protocol, patients can decide the surgery date for themselves because there is no need to wait for a leveling and complete decompensation of the arches ${ }^{11}$. In addition, this first phase of the conventional orthosurgical protocol is often difficult and unpleasant for the patient who sometimes wants to give up treatment.

Facial and functional improvements that the patient notices with the SFA are motivating and therefore helps to avoid a possible withdrawal from treatment before the surgery.

One of the other major benefits of this protocol is the postoperative orthodontic movement of teeth at a much faster rate, which decreases the overall duration of treatment ${ }^{14}$. For example, RAP has been the main reason for the surgery first protocol for many years: Postoperative orthodontic movements are faster, easier, and more accurate. The different osteotomies performed during surgery create biological activa- tion with increased blood flow within the alveolar bone due to the healing process. This phenomenon facilitates the dental movements in maxillary bases $5,16,21,22,23$.

It was described as early as 1977 by Epker and Fish ${ }^{9}$ almost 24 years before the RAP study in the orthodontic treatment by Wilcko et al. ${ }^{40}$. In fact, they claim that the increase in cell turnover and bone remodeling throughout the surgical area of the bone results in an accelerated postoperative orthodontic movement. In 2001, Wilcko et al. showed that the effect of corticotomies was accelerated bone turnover and a regional decrease in bone density facilitating tooth movement. The same biological effects seem to occur after orthognathic surgery resulting in accelerated movement during orthodontic treatment ${ }^{40}$. Liu et al. in $2011^{23}$ hypothesized that postoperative orthodontic movement acceleration could be related to increased osteoclastic activity and metabolic changes in the dentoalveolar bone for 3-4 months after surgery, which may then induce accelerated tooth movement.

This acceleration of tooth movements after surgery is also because of the restoration of a normal functional and anatomical relationship between the bones and surrounding soft tissues allowing more efficient tooth movements and easier orthodontic decompensation 2,14. Lee also supported this argument in 1994, stating that orthodontic treatment is easier to perform after early establishment of a normal skeletal and soft-tissue environment through orthognathic surgery. In effect, it eliminates soft-tissue resistance to dental decompensation movements ${ }^{19}$. This change improves the tone of the upper lip and provides 
a better position of the tongue, which increases the efficiency of incisive decompensation thanks to the muscular forces applied to the two arches. This early muscle adaptation helps the movement of the teeth, because it goes in the same direction, and thus stabilizes this orthodontic decompensation ${ }^{26}$.
Moreover, the movement of the teeth can be easily obtained because there is generally no more dental work. Huang et al. ${ }^{14}$ have observed more favorable biological tooth movements and more predictable occlusal results with better coordination of the upper and lower dental arches.

\section{RESULTS COMPARED TO A CONVENTIONAL PROTOCOL}

Numerous studies have highlighted the prolonged overall duration of treatments using conventional protocols, especially the long phase of preoperative orthodontics $7,24,25,27,33$, which has not necessarily been very effective ${ }^{2,31}$. It is a time-consuming process that can take up to 24 months with a median duration of 15-17 months depending on the studies $8,24,25,30$. With a SFA, the total duration of treatment is usually shorter, which has helped to make this approach more attractive to patients, surgeons, and orthodontists ${ }^{36}$. In fact, most of the surgery first studies have described postoperative orthodontic treatments of about 1 year ${ }^{2,21,26,39}$ or 6-9 months ${ }^{12,34,38 \text {, }}$ which is 6-12 months shorter compared to the conventional approach ${ }^{11,22}$. This decreased treatment time could then prevent complications such as the appearance of caries, gingival recessions, and root resorptions often observed following long orthodontic treatments ${ }^{2}$. It is important to note that the various factors such as the rapid restoration of normal functions and anatomic muscle relationships, as well as the absence of strong occlusal contacts, contribute to decreasing the total treatment time ${ }^{26}$.

On the other hand, different authors have compared the results of treat- ments of patients treated with both approaches. Liao et al. ${ }^{21}$ concluded that both groups had similar treatment outcomes for facial, occlusion, and stability, but with a shorter treatment time in the surgery first group. Ko et al. ${ }^{18}$ also concluded that the end results did not differ between the two approaches in terms of skeletal correction. Therefore, if the existing dental compensations achieve the goals of orthognathic surgery, a long preoperative orthodontic period is unnecessary.

We conducted a preliminary study whose objective was to evaluate the treatment results of surgery first patients with a total treatment time $\leq 6$ months using the objective graduation system (OGS) of the American Board of Orthodontists (ABO).

This retrospective study was conducted in a private practice in Medellin, Colombia, where the surgery first protocol is used routinely. The eight criteria of the ABO OGS index were measured: alignment, marginal ridges, vestibulolingual inclination, occlusal contacts, occlusal relationships, overhang, interdental contact points, and root angulation (Table 1, Fig. 5). The results were then compared with those of other studies with the same index of orthosurgical cases 
Table I: OGS score by criterion.

\begin{tabular}{|l|l|l|l|l|}
\hline & Average & SD & SE & Range \\
\hline Alignment & 12.6 & 3.5 & 1.4 & $9.5-19.5$ \\
\hline Marginal ridges & 4.3 & 2.1 & 0.8 & $2-8.5$ \\
\hline VL inclination & 5.5 & 3.9 & 1.5 & $1.5-13$ \\
\hline Overhang & 3.5 & 2.1 & 0.8 & $1.5-8$ \\
\hline Occlusal contacts & 2.9 & 3.2 & 1.3 & $0.5-9.5$ \\
\hline Occlusal relations & 4 & 3.3 & 1.3 & $0-9$ \\
\hline Interproximal contacts & 0 & 0 & 0 & $0-0$ \\
\hline Root angulation & 1.5 & 1.1 & 0.4 & $0-3$ \\
\hline Total & 34.5 & 11.2 & 4.5 & $22-48.5$ \\
\hline
\end{tabular}

SCORE OGS 0-20

SCORE OGS 21-30

SCORE OGS $>30$

Figure 5

Percentage of subjects according to OGS score.

performed with a conventional protocol. We came to the same conclusion as the previous authors, patients treated with the SFA with a short duration of treatment ( $\leq 6$ months) seem to show results equal to those of patients treated conventionally.

Finally, different studies ${ }^{18,21,36,39}$ evaluated the stability of craniofacial struc-

\section{CLINICAL CASE}

Katerina, 28 years of age, presented for a consultation at the clinic for significant facial asymmetry associated with mandibular prognathism. We observed a mandibular deviation to the right and tures at least 1 year after orthognathic surgery comparing the approach of surgery first and the conventional approach. In view of the conclusions of these studies, the long-term results of the surgery first protocol show that the transverse, vertical, and sagittal dimensions of dental and skeletal stability are similar or even better. an inverted anterior malocclusion are observed (Figure 6).

A 6-mm mandibular retraction was simulated on models mounted on an 

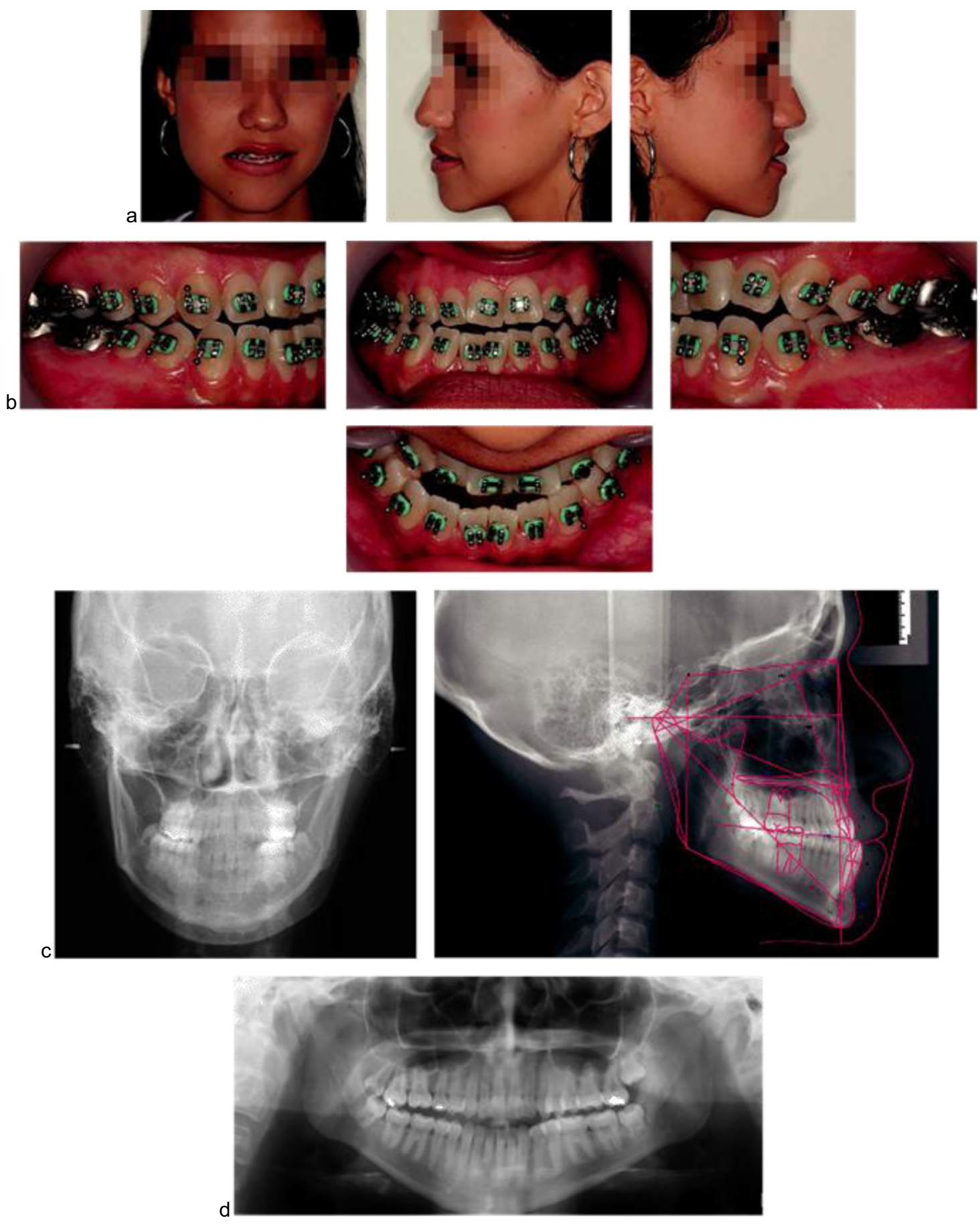

Figure 6

Start of treatment documents. (a) Extraoral photographs. (b) Intraoral photographs. (c) Teleradiography of face and profile. (d) Panoramic x-ray. 

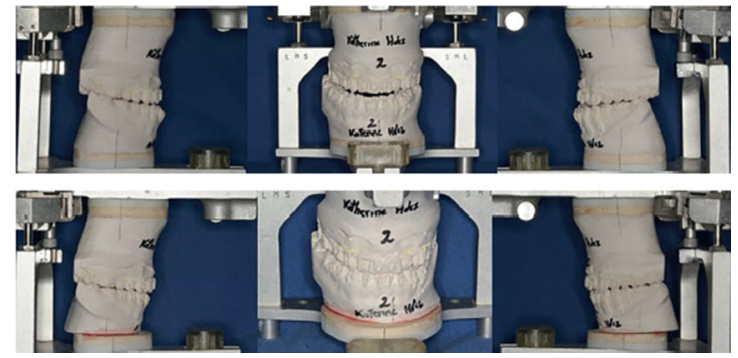

Figure 7

Surgical planning on articulator mounted models. Initial occlusal relationships. Occlusal relations after simulation of mandibular retraction.

articulator, it will be performed along with genioplasty (Figure 7).

Miniplates were surgically placed at the same time to achieve a retraction of the maxillary lateral sectors allowing the correction of the anterior maxillary congestion without increasing the overhang (Figure 8).

\section{CONCLUSION}

Despite the many challenges of performing orthognathic surgery before orthodontic decompensation, the multiple benefits of this approach and the results of treatment obtained show the need to incorporate the surgery first protocol into standard treatment options for orthosurgical cases. In fact, the decrease in the duration of treatment as well as the increase in patient satisfaction and cooperation prioritize the patient's well-being and demand, without compromising the quality and stability of the end-of-treatment results.
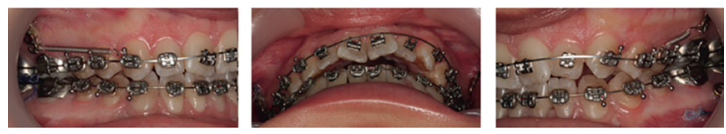

Figure 8

Retraction of maxillary lateral sectors on anchor plates.
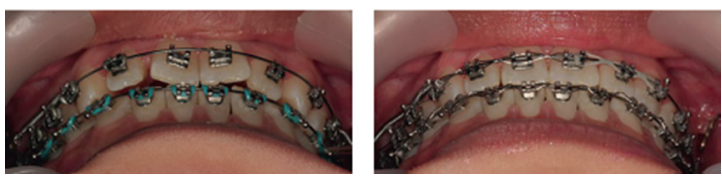

Figure 9

Alignment of maxillary incisors and correct overhang (5 months).

In 5 months, the space needed to align the incisors and obtain a correct overhang were obtained (Figure 9).

Surgical and orthodontic treatment was performed in 7 months, which resulted in a very satisfactory esthetic result, a correction of facial asymmetry and Skeletal Class III, as well as functional occlusal ratios (Fig. 10).

With a focus on case selection, treatment planning, and two-way exchange between the surgeon and the orthodontist, the SFA achieves a high level of patient and clinician satisfaction. It is therefore a change of perspective that the clinician must adopt to integrate this new effective and advantageous treatment model into their therapies.

Illustrations: Courtesy of Dr. Carlos Villegas, Medellin, Colombia.

Conflict of interest: The author declares that there is no conflict of interest. 

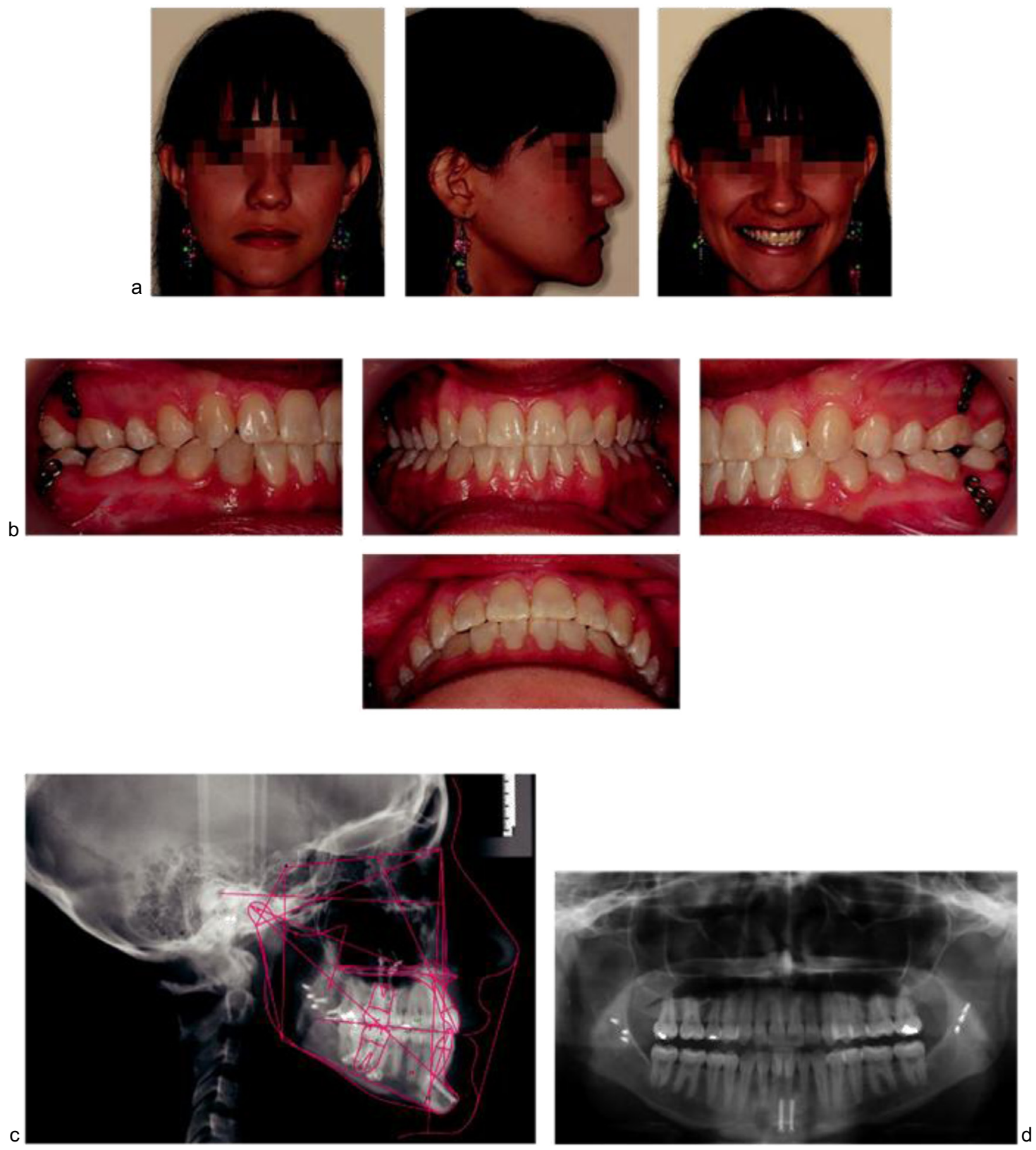

Figure 10

End-of-treatment documents (7 months). a) Extraoral photographs. b) Intraoral photographs.

c) Teleradiography in profile. d) Panoramic X-ray. 


\section{BIBLIOGRAPHY}

1. Aristizábal JF., Martínez Smit R, Villegas C. The 'surgery first' approach with passive self-ligating brackets for the treatment of skeletal Class III malocclusion. J Clin Orthod 2015;49:361-370.

2. Baek HS, HW, Kwon YH, Choi JY Ahn. Surgery-first approach in skeletal class III malocclusion treated with 2-jaw surgery: evaluation of surgical movement and postoperative orthodontic treatment. J Craniofac Surg 2010;21:332-338.

3. Behrman SJ, Behrman DA. Oral surgeons' con siderations in surgical orthodontic treatment. Dent Clin North Am 1988;32:481-507.

4. WH Bell. The strong I osteotomy for correction of $n$. deformities. J Oral Surg 1975;33: 412-426.

5. Bell W. Accelerated orthognathic surgery and increased orthodontic efficiency: a paradigm shift. J Oral Maxillofac Surg 2011;579-583.

6. Caldwell JB, Letterman GS. Vertical osteotomy in the mandibular ramli for correction of prognathism. J Oral Surg 1954;185-202.

7. Diaz PM. et al. Time used for orthodontic surgical treatment of dentofacial deformities in white patients J Oral Maxillofac Surg 2010;68:88-92.

8. Dowling PA, L Espeland, Krogstad O, Stenvik, Kelly A. Duration of orthodontic treatment involving orthognathic surgery. Int J Adult Orthodon Orthognath Surg 1999;14:146-152.

9. Epker BN, Fish L. Surgical-orthodontic correction of open-bite deformity. Am J Orthod 1977;71:278-299.

10. Epker BN, Wolford LM. Middle-third facial osteoto-mies: their use in the correction of acquired and deve lopmental dentofacial and craniofacial deformities. J Oral Surg 1975;33:491-514.

11. Hernández-Alfaro F, Guijarro-Martínez R. Defi-nition of the appropriate timing for surgical intervention in orthognathic surgery. Int J Oral Maxillofac Surg 2014;43:846-855.

12. Hernández-Alfaro F, Guijarro-Martínez R, Molina-Coral A, Badía-Escriche C. 'Surgery first' in bima xillary orthognathic surgery. J Oral Maxillofac Surg 2011;69: e201-207.

13. Hernández-Alfaro F, Guijarro-Martínez R, Peiró-Guijarro MA. Surgery first in orthognathic surgery: what have we learned? A comprehensive workflow based on 45 consecutive cases. J Oral Maxillofac Surg 2014;72:376-390.

14. Huang CS, Hsu SS, Chen YR. Systematic review of the surgery-first approach in orthognathic surgery. Biomed J 2014;37:184-190.

15. Hyon WS, Park S, Lee YWS. Surgery-First-Orthognathic Approach (SFOA) to Prognathism: Indications and Limitations. J Oral Maxillofac Surg 2008;66:39-40.

16. Kim JH, Mahdavie NN, Evans CA. In: Orthodontics - basic aspects and clinical considerations 265-300 (Bourzgui F, 2012).

17. Kim JMNE. Guidelines for 'Surgery First' Orthodontic Treatment. Orthodontics - Basic Aspects and Clinical Considerations, 2012.

18. Ko EW, Hsu SS, HY Hsieh, YC Wang, Huang CS, Chen YR. Comparison of progressive cephalometric changes and postsurgical stability of skeletal Class III correction with and without presurgical orthodontic treatment. J Oral Maxillofac Surg 2011;69:1469-1477.

19. Lee RT. The benefits of post-surgical orthodontic treat - ment. Br J Orthod 1994;21: 265-274. 
20. Leelasinjaroen P, Godfrey K, Manosudprasit M, Wangsrimongkol T, Surakunprapha P, Pisek P. Surgery first orthognathic approach for skeletal Class III malocclusion corrections - a literature review. J Med Assoc Thai 2012;95 Suppl 11, S172-180.

21. Liao YF, Chiu YT, Huang CS, EWC Ko, Chen YR. Presurgical orthodontics versus no presurgical ortho-dontics: treatment outcome of surgical-orthodontic correction for skeletal Class III open bite. Plast Reconstr Surg 2010;126:2074-2083.

22. Liou EJ, Chen PH, Wang YC, Yu CC, Huang CS, Chen YR Surgery-first accelerated orthognathic surgery: orthodontic guidelines and setup for model surgery. J Oral Maxillofac Surg 2011;69:771-780.

23. Liou EJ, Chen PH, Wang YC, Yu CC, Huang CS, Chen YR. Surgery-first accelerated orthognathic sur-gery: postoperative rapid orthodontic tooth move-ment. J Oral Maxillofac Surg 2011;69:781-785.

24. Luther F, Morris DO, Hart C. Orthodontic preparation for orthognathic surgery: How long does it take and why? A retrospective study. BR J Oral Maxillofac Surg 2003;41:401-406.

25. Luther F, Morris $C$, Karnezi K. Orthodontic treatment following orthognathic surgery: how long does it take and why? A retrospective study. J Oral Maxillofac Surg 2007;65: 1969-1976.

26. Nagasaka H, Sugawara J, Kawamura, h., Nanda a. 'Surgery first' skeletal Class III correction using the Skeletal Anchorage System. J Clin Orthod 2009;43:97-105.

27. O'Brien K. et al. Prospective, multi-center study of the effectiveness of orthodontic/orthognathic surgery care in the United Kingdom. Am J Orthod Dentofac Orthop 2009;135: 709-714.

28. Obwegeser HL. Mandibular Growth Anomalies: Terminology - Aetiology Diagnosis - Treatment. Springer Science \& Business Media, 2013.

29. Pedraza R, JC Serna, Juri JB. Surgery First approach in skeletal Class III with condylar Hyperplasia. ACTA Otorrilolaringologia y cirugia cabez y cuello 2013;147-152.

30. Proffit WR, Miguel JA. The duration and sequencing of surgical-orthodontic treatment. Int J Adult Orthodon Orthognath Surg 1995;10:35-42.

31. Proffit WR, White RP. In: Contemporary Treatment of Dentofacial Deformity 245-267, Proffit WR, RP White, DM Sarver, 2003.

32. Poulton DR, Taylor RC, Ware WH. Cephalometric x-ray evaluation of the vertical osteotomy correction of man-dibular prognathism. Oral Oral Surg Med Oral Pathol 1963;16:807-820.

33. Slavnic S, Marcusson A. Duration of orthodontic treatment in conjunction with orthognathic surgery. Swed Dent J 2010;34;159-166.

34. Sugawara J, Aymach Z, Nagasaka DH, Kawamura H, Nanda a. 'Surgery first' orthognathics correct to a skeletal Class II malocclusion with year impinging Dick. J Orthod 2010 blink;44:429-438.

35. Trauner $\mathrm{R}$, Obwegeser $\mathrm{H}$. The surgical correction of mandibular prognathism and retrognathia with con-sideration of genioplasty. I. Surgical procedures to correct mandibular prognathism and reshaping of the chin. Oral Oral Surg Med Oral Pathol 1957;10:677-689; contd.

36. Uribe F. et al. Treatment duration and factors asso-ciated with the surgery-first approach: a two-center study. Prog Orthod 2015;16:29.

37. Villegas $C$, Jacob $N$, Uribe F, Nanda a. Rotation of the maxillomandibular complex to enhance aesthetics using tics has 'surgery first' approach. J Orthod 2012;46, 85-91 blink. 
38. Villegas C, Uribe F, Sugawara J, Nanda a. Expedited correction of significant asymmetry using dentofacial has 'surgery first' approach. J Clin Orthod 2010;44:97-103.

39. Wang YC, Ko EW, Chen Huang CS, Yan Chen, Takano-Yamamoto T. Comparison of transverse dimensional changes in skeletal Class III surgical patients with and without presurgical orthodontics. J Oral Maxillofac Surg 2010;68:1807-1812. 